\title{
Innovative pupil topographies for sparse aperture telescopes and SNR
}

\section{James Breckinridge, Nevin Bryant, John Lorre}

James Breckinridge, Nevin Bryant, John Lorre, "Innovative pupil topographies for sparse aperture telescopes and SNR," Proc. SPIE 7013, Optical and Infrared Interferometry, $70133 E$ (28 July 2008); doi: 10.1117/12.787011

SPIE Event: SPIE Astronomical Telescopes + Instrumentation, 2008, Marseille, France 


\title{
Innovative pupil topographies for sparse aperture telescopes and SNR
}

\author{
James Breckinridge, Nevin Bryant, and John Lorre $\dagger$ \\ Jet Propulsion Laboratory, California Institute of Technology \\ 4800 Oak Grove Drive, Pasadena, California 91109
}

\begin{abstract}
Since the first application of the telescope to astronomy in 1610, most new astronomical discoveries require larger and larger radiation collecting areas. Today, the twin 10-meter Keck telescopes are operational and several 30-meter-aperture class telescopes are being planned. Optical interferometers and sparse aperture ground telescopes for astronomy have been proposed and built. Fienup showed the dependence between exposure time and the dilution factor of the aperture needed to maintain image quality. ${ }^{1}$ Carpenter suggests a sparse aperture telescope system for the purpose of imaging across the surfaces of stars. ${ }^{2}$ This paper demonstrates that the ability to reconstruct images from white-light extended sources with different contrast levels also depends on the specific pupil topography that is applied to the telescope system. Signal-to-noise ratios for recorded images are calculated for scene contrast, pupil shape, detector full-well, detected photons, and exposure times.
\end{abstract}

Keywords: telescopes, sparse apertures, image quality, signal-to-noise ratio, image processing

\section{INTRODUCTION}

Historically, telescopes for astronomical research have used a monolithic surface to collect the incoming radiation. The larger the telescope aperture, the more photons captured and the fainter the object recorded. In addition, as the telescope aperture becomes larger, the angular resolution increases. Primary mirror mass grows not only because of the increased aperture, but the fundamental need to maintain the stiffness of the mirror so there is a coherent white-light wavefront reflected from the large mirror surface. The mass of the classical telescope monolithic mirror increases with the cube of the diameter and quickly becomes too big to be cost effective and practical. Light-weighting telescope mirrors, that is removing mass from the structure while maintaining the required stiffness, has been an area of intense technological development over the past few years. Developments in high-speed micro-electronics and small devices have enabled lightweight, very fast electro-mechanical servo-control systems, which have found applications in both large telescopes as well as temporal and spatial interferometers. These control systems today are regularly applied to large telescopes to create a stiff primary mirror using reference lasers and control systems.

Innovative optical designs are implemented using "two-stage" optics ${ }^{3,4}$ to correct for the aberration in a large telescope mirror that is lightweight, and therefore of reduced stiffness. Some astronomical and planetary space observing needs require high angular resolution on relatively bright sources. This leads to a consideration of sparse aperture telescope systems. Image quality from sparse aperture optical telescopes has been extensively studied by Fiete ${ }^{5}$ and Fienup. ${ }^{1}$

This paper assesses the signal-to-noise ratio (SNR) for sparse aperture telescopes recording white-light extended scenes of several different contrasts, discusses SNR for sparsely filled scenes, and examines the ability of sparse aperture telescope systems to image low contrast complex scenes with different pupil topographies.

To better constrain the general dimensions of different sparse aperture topographies, numerically simulated sparse aperture imagery was used. The following issues were anticipated to impact image quality:

1. Scene contrast (which restricts SNR)

2. Pupil shape and its resulting optical transfer function

3. Frequency $(\mathrm{U}-\mathrm{V})$ domain coverage

\footnotetext{
* The majority of the work documented here was completed before 1999.

${ }^{\dagger}$ Deceased
} 

4. Spectral bandwidth
5. Phasing errors between primary aperture mirror elements
6. Detector full-well
7. Exposure time

Each of these factors was investigated as independently as possible. This paper demonstrates that the scene contrast is a significant constraint. Sparse systems perform poorly at low contrast, resulting in a tradeoff between sparseness, number of detected photons (dp), and exposure time. This paper also shows that pupil shape and fill factor are important constraints on image quality. The optimum pupils for high-resolution Earth imaging contain an outer ring of elements, have smooth transfer functions, and have fill factors that exceed $30 \%$.

\section{BACKGROUND}

Aden and Marjorie Meinel discuss the engineering merits of sparse aperture telescopes for space astronomy. ${ }^{6}$ They investigate the system practicality to show that sparse aperture telescopes may be realized in the future.

\subsection{SNR for white-light broadband imaging with sparse aperture telescopes}

Astronomers recognized in the mid-1950s that an expensive single large aperture telescope could be divided into a series of less expensive smaller apertures coherently interconnected to provide nearly the same performance as that of the large aperture. The series of smaller apertures, however, required more extensive ground data processing to reconstruct an equivalent image.

Radio astronomers and electrical engineers were the first to develop innovative image reconstruction techniques using sparse apertures and then synthesize the radio-image scene. The Very Large Array (VLA), a radio telescope, is an example. The radio astronomers applied image processing algorithms to optical scenes, and extrapolated their calculations to suggest that sparse aperture optical telescopes are useful for imaging optical sources with optical telescopes. These extrapolations are not entirely correct.

At radio waves, the noise temperature of the receiver, sometimes called the antenna temperature, dominates the signalto-noise ratio. In the UV, optical and near-infrared region of the spectrum, the signal-to-noise ratio (SNR) is dominated by the signal-photon arrival rates, which are established by the nature (i.e., color and brightness) of the source and not the receiver(s). In the radio region of the spectrum, both amplitude and phase of the received signal are recorded at optical frequencies; in white light (where a heterodyne process cannot be used), the phase cannot be directly recorded.

This paper examines the limitations to the sparse-aperture imaging broadband white-light spatially extended optical scenes containing a variety of contrast information. Fienup showed that the exposure time increases between the 2.5 and 3rd power of the aperture fill factor to achieve the same SNR as that for a filled aperture. We show that the value of the exponent also depends on the topology of the telescope aperture, scene contrast, and number of detected photons.

\section{METHODOLOGY}

\subsection{Computer modeling of several sparse aperture telescopes}

Several pupil topographies were modeled and applied to a simulated white-light extended scene to understand how noise propagates through the sparse aperture image formation process. Comparisons were then made between six pupil topographies with the same scene containing 1,000, 4,000, and 40,000 detected photons (dp).

Figure 1 shows six pupils in the lower row with their companion log optical transfer functions (OTF) across the top row. Six apertures, shown left to right, in Figure 1 were selected for comparison - a filled pupil (monolith), an annulus, a necklace of 32 touching circular mirror segments, a gap necklace of 17 spaced circles, a quasi-non-redundant set of 21 circles, and a VLA "Y." Column 1 shows a filled aperture telescope; column 2, an annulus with the same resolution of the filled aperture and a fill factor of 0.2 ; column 3, a necklace of 32 touching circles with the same resolution as the filled aperture and a fill factor of 0.2 ; column 4 , a gap necklace of 17 spaced circles with the same resolution as the filled aperture and a fill factor of 0.2 ; column 5, a quasi-non-redundant arrangement of 21 circles in three rings with the same resolution of the filled aperture and a fill factor of 0.2 ; and column 6 , a VLA "Y" configuration with large redundancy. 


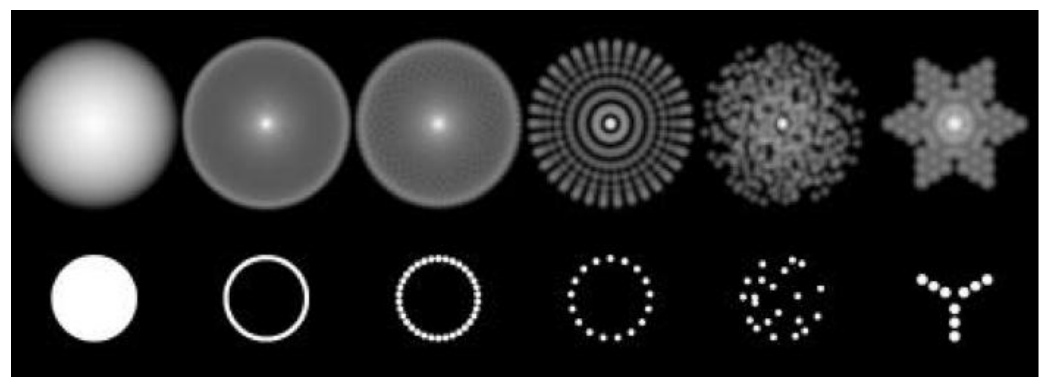

Figure 1. Six pupils with $\log$ optical transfer functions and point spread functions

Figure 2 uses a single image obtained in the colors red, green, blue (RGB) of the San Francisco Presidio at 50-cm resolution from a low-flying aircraft as the input scene. The apparent angular size of the pupil is, of course, dependant on wavelength. These RGB images were converted to gray scale for much of the work discussed here. Section 4.4 below provides a brief discussion of the chromatic effects on image reconstruction and image quality. Contrast was varied for different copies of this gray-scale image and the pupil topography changed. Images were generated in three blocks, each corresponding to a different number of detected photons. Each block has two paired columns and six rows of images, each of which represents a different pupil. The pupils are shown in the same order as the image rows. The right column is the raw image as created by the aperture in question. The left column is the image as processed using a Wiener deconvolution technique on the image to its right. In each case, the amount of restoration was selected to minimize the difference between the restoration and the reference or original image. For those images in column 1, the source scene had 1,000 dp; for those images in column 2, the source scene had 4,000 dp; and for those images in column 3, the source image had 40,000 dp.
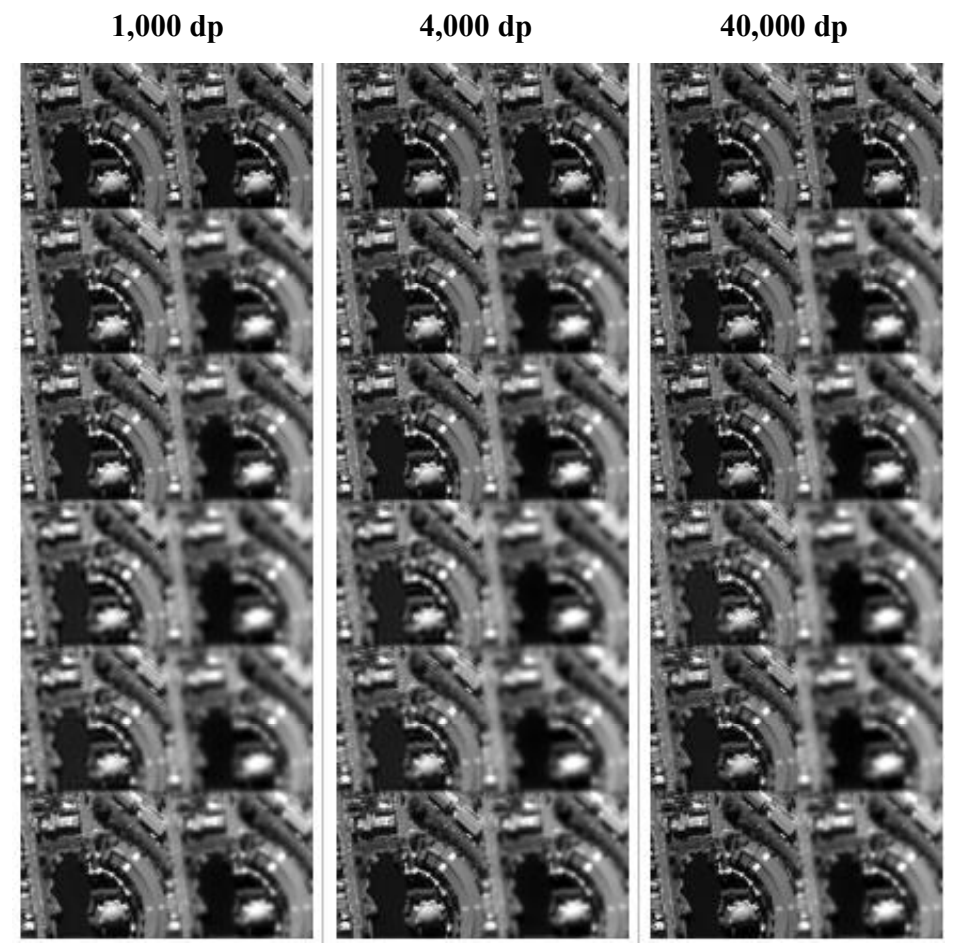

Filled

Annulus

Touching Necklace

Gap Necklace

Quasi-non-redundant

VLA "Y"

Figure 2. Thirty six images were created from a single image looking directly down onto a building at the San Francisco Presidio. Six rows, each corresponding to a different pupil topography given in Figure 1, are pairs of images recorded at $1,000,4,000$, and 40,000 detected photons. The image to the right in each pair is a simulated recording of the whitelight image and to the left is the Weiner-filtered optimized image reconstruction. The differences between many of the images shown above are difficult to see. Quantitative analysis was used for the conclusions later. 
A merit function was developed to compare the before and after images. We arrived at the following conclusions:

1. $1,000 \mathrm{dp}$ may be adequate for a filled pupil, but much larger numbers of dp are required to permit deconvolution for sparse apertures. For example, the annulus at $40,000 \mathrm{dp}$ is equivalent in quality to the filled pupil at only $1,000 \mathrm{dp}$.

2. Pupils that have holes in U-V coverage at low spatial frequencies seem incapable of producing quality imagery. Note that the images for the 17-element necklace are very poor in quality.

3. Nearly non-redundant pupils with OTF that are very low at some regions within the pupil produce poor images in the presence of noise. Compare the images of the 21 quasi-non-redundant pupils in column 5 of Figure 1 to the 1,000-dp and the 40,000-dp cases. This failure is due to random noise overwhelming the spatial frequency information located at low OTF. Its effect is to mimic a pupil with inadequate U-V coverage.

4. An annulus can be mimicked by a string of circles.

\subsection{Integration time and scene contrast: CCD full-well limits and exposure limits at three contrast levels}

This section investigates the ability of sparse aperture telescopes to image low contrast scenes, and the limitations imposed by deconvolution in the presence of shot noise. Charge coupled devices (CCD) are limited to full-wells of approximately 100,000 dp. We considered exposure times to be around 1/30 second. We computed the full-well and exposure times required for various sparse configurations to replicate the quality of an image acquired by a filled aperture. We then computed the dp rate for a filled circular aperture. By selecting the number of dp and the contrast of a scene, the physical exposure time in seconds was determined. Since we desired the sparse apertures to match the image quality of this filled aperture, we were then able to determine the physical exposure time for the sparse apertures as well. All of this was based upon the assumption that $6,000 \mathrm{dp}$ per pixel were adequate for a filled aperture operating upon a scene with $10 \%$ contrast. This image was barely acceptable, providing a lower limit on the number of dp required for sparse pupils. The imagery could be improved, but at the expense of increasing the severity of the requirements on the sparse configurations. This was tested by using $24,000 \mathrm{dp}$ in place of the of $6,000 \mathrm{dp}$ used for $10 \%$ contrast imagery. The result was that none of the sparse pupils presented in this report could provide useful imagery.

The selected image of the Presidio in San Francisco was reduced to $20 \%, 10 \%$, and $5 \%$ contrast, providing the three images used in this study. In order to relate the three contrast tests, the numbers of dp were selected for the filled reference aperture, which produced the same RMS error in the image. 1,300 dp was used at $20 \%$ contrast, $6,000 \mathrm{dp}$ at $10 \%$ contrast, and $23,400 \mathrm{dp}$ at $5 \%$ contrast. This ensured that the three filled aperture images $(20 \%, 10 \%, 5 \%)$ were identical and that the same RMS image quality for all pupils were applied.

This progression of $\mathrm{dp}$ as a function of image contrast led to the computation of dp required for a $2 \%$ contrast scene. The result was $135,000 \mathrm{dp}$, a value near full-well. This result implies that at low contrasts of $2 \%$ or less, even a filled aperture is unable to generate acceptable image quality, leaving no room for any sparse configuration. Therefore, a rate of $2 \times 10^{6}$ $\mathrm{dp} / \mathrm{second} / \mathrm{pixel}$ was used in this study.

Note that these assumptions of a physical optical system only constrain the physical exposure time in seconds, not the numbers of dp required to achieve a certain deconvolved image quality. If, for example, the $\mathrm{dp}$ rate was halved due to selecting a different optical configuration, the exposure times in seconds would double but none of the other tabulated values computed in this study would change.

One might ask if the difference in exposures between sparse and filled apertures should only depend on the degree of sparseness in the sparse configuration. It turns out that sparseness is only one factor. The overriding factor is the ability to deconvolve the sparse image in the presence of noise. Each pupil has its own characteristics, best described by its OTF. When the OTF becomes low, noise corrupts those spatial frequencies, requiring a higher than expected signal-tonoise ratio to successfully deconvolve the image. In order to solve this primary problem, a combination of both a "cooperative" pupil and a reasonable fill factor is required.

\subsection{Method of determining relative exposure times by matching the RMS residual from a filled aperture with that of a sparse aperture}

The method central to this analysis enables one to determine the exposure required by a sparse aperture relative to a filled aperture. It is based upon finding the number of dp required by some sparse aperture, which gives a deconvolved 
image whose RMS residual (difference between a perfect reconstruction and the one achieved) is the same as that achieved by a filled aperture. The challenge is, at each estimate of the required number of photons, to determine the optimal Wiener filter, which deconvolves the image optimally, i.e., in an RMS sense. To accomplish this, the simulator minimizes a function (the RMS residual) based upon a single parameter, which is the Wiener gain constant $G$. Ideally $G^{2}$ is the ratio of the power spectrum of the signal to the power spectrum of the noise. In practice, these parameters are unknown but can be approximated by a constant at high spatial frequencies.

$$
\text { Deconvolution filter }=\frac{O T F^{*}}{|O T F|^{2}+1 / G^{2}}
$$

The steps required to determine relative exposure times between sparse and filled apertures are listed below:

1. Convolve a test image with a filled aperture without noise and deconvolve it using a Wiener filter with a gain of 100. This produces a clean-band, limited reference image. Call this image $\boldsymbol{R}$.

2. Convolve the test image with a filled aperture, using $\boldsymbol{M} \mathrm{dp} / \mathrm{pixel}$ as the average of noise, then deconvolve it using the optimal Wiener filter using the image $\boldsymbol{R}$ as a reference. Call this new image $\boldsymbol{F}$. The optimal filter is the gain factor that minimizes the difference between $\boldsymbol{F}$ and $\boldsymbol{R}$.

3. Calculate the RMS difference between the images $\boldsymbol{R}$ and $\boldsymbol{F}$. We refer to this as $\boldsymbol{D}$ 1.

4. Convolve the test image (from step 1) with one of the sparse pupils whose fill factor is $\boldsymbol{S}$ and introduce $\boldsymbol{N}$ $\mathrm{dp} /$ pixel of noise.

5. Deconvolve this image using an optimal Wiener filter. We refer to this deconvolved image as $\boldsymbol{X}$.

6. $\quad$ Calculate the RMS difference between $\boldsymbol{R}$ and $\boldsymbol{X}$. We refer to this as $\boldsymbol{D} 2$.

7. Iterate steps 4,5 , and 6 , trying different numbers of dp/pixel of noise, $N$ until $D 1 \cong D 2$.

8. The exposure of the sparse pupil relative to the filled pupil is then:

$$
\left[\frac{N}{M}\right] \cdot \frac{1}{S}
$$

9. Compare the images $\mathrm{X}$ and $\mathrm{F}$. The values should be nearly identical.

\subsection{Rationale for selecting the pupils used in this analysis}

As seen in previous studies, systems with "bumpy" OTFs performed poorly in broadband applications. This analysis addressed this problem by introducing mirrors of different sizes. The 3-and 5-arm Ys used had small mirrors in the center, which increased in size and spacing outward along the branches. This greatly smoothed the OTF. Combining a 5arm Y with a necklace produced a good OTF, which was cone-shaped similar to the modulation transfer function (MTF) of a filled aperture and nearly featureless. Recall that the OTF has a real and imaginary (amplitude and phase) term and that $\mathrm{MTF}=|\mathrm{OTF}|^{2}$. This configuration produced the finest imagery of any sparse aperture pupil we have tested to date.

\section{RESULTS}

\subsection{Results from processing a 20-percent contrast image}

Figure 3 shows six pupils in the lower row with their companion log optical transfer functions (OTF) across the top row. From left to right, the six pupil topographies are identified as filled, touching necklace, necklace $+5 \mathrm{arms}, 5 \mathrm{arms}, 3 \mathrm{arms}$ (Y), and Golay-12. These were used to process images whose contrast was reduced to $20 \%$. 


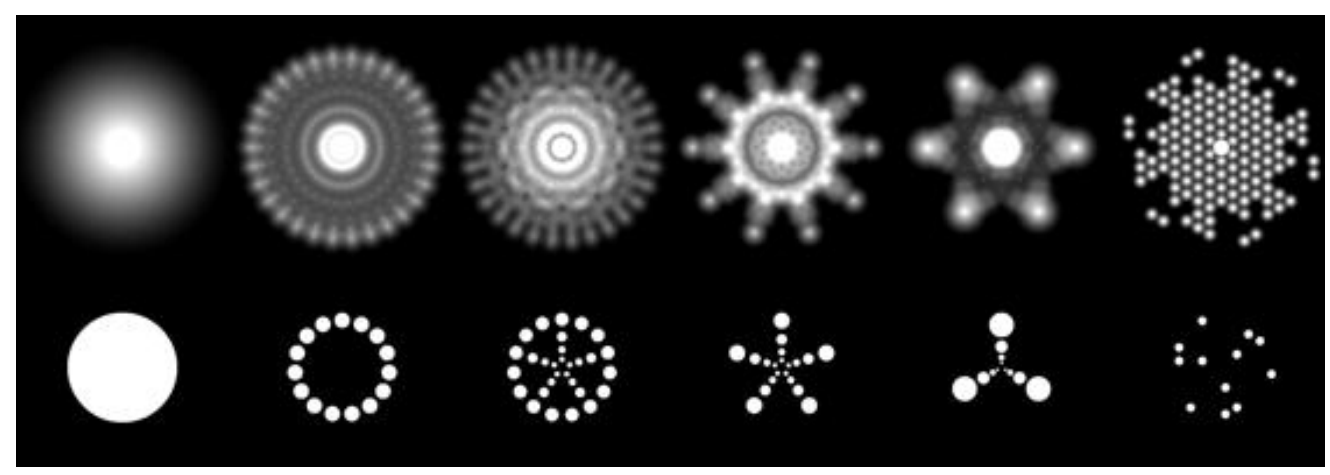

Figure 3 . The $20 \%$ contrast image was processed using each of these six pupil topographies. Note that these are different than the pupil topologies used in the earlier section. This was done to gain an intuitive understanding of the effects of the pupil arrangement on image quality, contrast, and signal to noise ratio (SNR). The six pupils are shown in the lower row with their companion log optical transfer functions (OTF) across the top row. Reading left to right, they are described as filled, touching necklace, necklace +5 arms, 5 arms, 3 arm (Y), and Golay-12.

As the baseline, we selected 6,000 dp per pixel for a 10\% contrast image viewed through a filled aperture. This produced a certain RMS residual, which was determined to be a limiting condition, with any poorer performance being unacceptable. To achieve the same residual, it was determined that $1,300 \mathrm{dp}$ per pixel was required for the $20 \%$ contrast case. The number of $\mathrm{dp}$ for sparse apertures was selected in order to match this RMS residual value (Table 1). The "exponent" term is the exponent $X$ in the relation:

$$
\text { Exposure }=\left(\frac{1}{\text { fill factor }}\right)^{X}
$$

Table 1 . Assuming the input scene has $20 \%$ contrast, this table provides a descriptor for the pupil topography; fill factor by percentage of the filled aperture (note that the outermost dimension of the pupil is held equal across all pupils); the number of detected photons required to achieve a reference exposure for a filled aperture; exposure time in seconds to collect the detected photons; and exponent, $X$, in Equation 2.

\begin{tabular}{|c|c|c|c|c|c|c|}
\hline Pupil & Description & $\begin{array}{c}\text { Fill Factor \% } \\
\text { by Area }\end{array}$ & $\begin{array}{c}\text { Detected } \\
\text { Photons } \\
\text { Required }\end{array}$ & $\begin{array}{c}\text { Exposure } \\
\text { Relative to a } \\
\text { Filled Aperture }\end{array}$ & $\begin{array}{c}\text { Exposure } \\
\text { Time (sec) }\end{array}$ & $\begin{array}{c}\text { Exponent } \\
\text { X }\end{array}$ \\
\hline 1 & Filled & 100 & 1,300 & 1 & 0.00065 & - \\
\hline 2 & $\begin{array}{c}\text { Touching } \\
\text { necklace }\end{array}$ & 29.6 & 90,000 & 234 & 0.152 & 4.48 \\
\hline 3 & $\begin{array}{c}\text { Necklace + } \\
\text { arms }\end{array}$ & 30.1 & 80,000 & 204 & 0.132 & 4.43 \\
\hline 4 & 5 arms & 18.6 & 108,000 & 446 & 0.290 & 3.63 \\
\hline 5 & 3 arms (Y) & 20.2 & 90,000 & 343 & 13.37 & 3.65 \\
\hline 6 & Golay-12 & 7.48 & $2,000,000$ & 20,568 & & 3.83 \\
\hline
\end{tabular}

\subsection{Results from processing a $10 \%$ contrast image}

Figure 4 shows six pupils in the lower row with their companion log OTF across the top row. From left to right, the six pupil topographies are identified as filled, touching necklace, necklace +5 arms, 5 arms, 3 arms (Y), and ring of $6+3$ arms. 


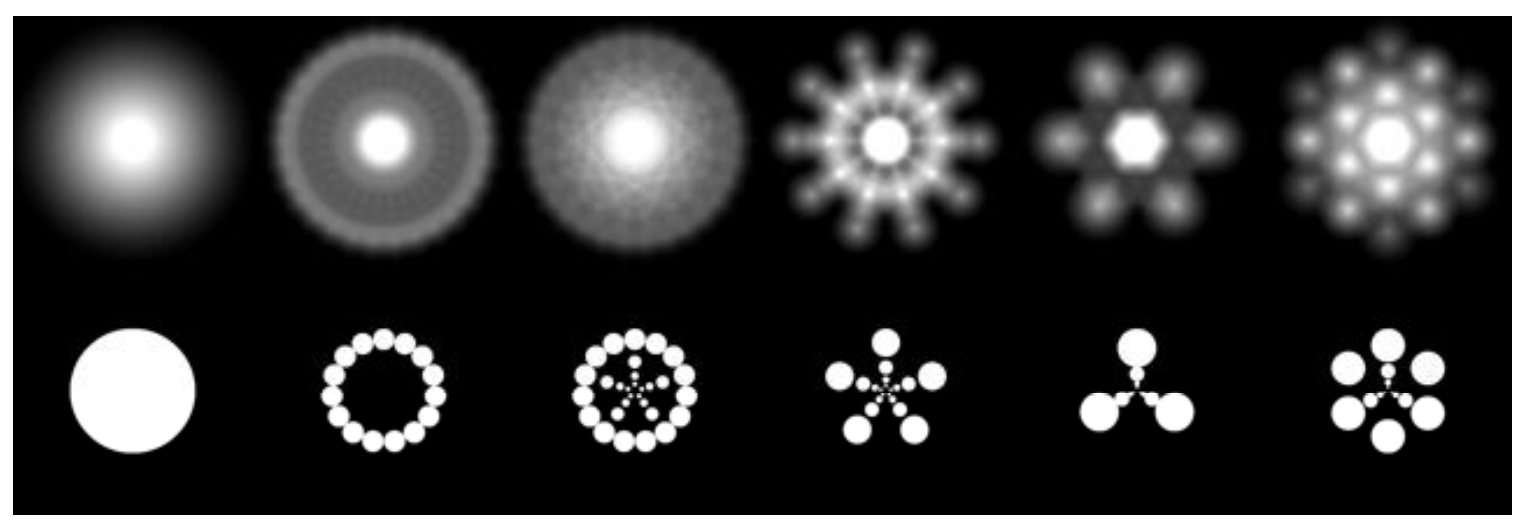

Figure 4. Six pupil topographies used to analyze the systems capability to record and process images with the $10 \%$ contrast level.

In this case, the number of dp per pixel was 6,000 for the filled aperture. The telescope systems response to recording the $10 \%$ contrast scene is given in Table 2 .

Table 2. The contents of this table summarize the results of our calculations for a $10 \%$ contrast input scene. The columns are a descriptor of the pupil topography; fill factor by percentage of the filled aperture (note that the outermost dimension of the pupil is held equal across all pupils); the number of detected photons required to achieve a reference exposure for a filled aperture; exposure time in seconds to collect the detected photons; and the calculated exponent, $X$, in Equation 2.

\begin{tabular}{|c|c|c|c|c|c|c|}
\hline Pupil & Description & $\begin{array}{c}\text { Fill Factor \% } \\
\text { by Area }\end{array}$ & $\begin{array}{c}\text { Detected } \\
\text { Photons } \\
\text { Required }\end{array}$ & $\begin{array}{c}\text { Exposure } \\
\text { Relative to a } \\
\text { Filled Aperture }\end{array}$ & $\begin{array}{c}\text { Exposure } \\
\text { Time (sec) }\end{array}$ & $\begin{array}{c}\text { Exponent } \\
\text { X }\end{array}$ \\
\hline 1 & Filled & 100 & 6,000 & 1 & 0.003 & -153 \\
\hline 2 & $\begin{array}{c}\text { Touching } \\
\text { necklace }\end{array}$ & 44.2 & 135,000 & 50.9 & 0.074 & 4.86 \\
\hline 3 & $\begin{array}{c}\text { Necklace + } \\
\text { arms }\end{array}$ & 51.7 & 76,500 & 24.7 & 0.215 & 3.93 \\
\hline 4 & 5 arms & 33.7 & 145,000 & 71.7 & 0.136 & 3.50 \\
\hline 5 & 3 arms (Y) & 33.6 & 92,000 & 45.6 & 0.061 & 4.15 \\
\hline 6 & $\begin{array}{c}\text { Ring of 6 +3 } \\
\text { arms }\end{array}$ & 48.5 & 59,000 & 20.3 & & \\
\hline
\end{tabular}

\subsection{Results from processing a $5 \%$ contrast image}

Figure 5 shows four pupils in the lower row with their companion log OTF across the top row. From left to right, the four pupil topographies are identified as filled, touching necklace, necklace +5 arms, and annulus. 


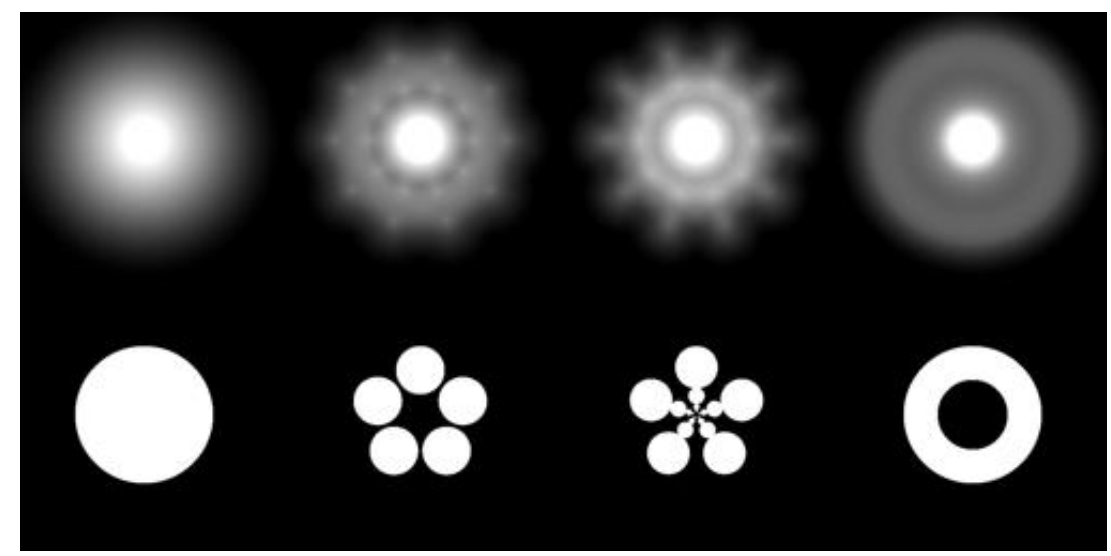

Figure 5. Four pupil topographies used to examine the systems ability to record and process images with the $5 \%$ contrast level.

In this case, the number of dp per pixel was 23,400 for the filled aperture. Table 3 summarizes our calculations of the performance of each of the four pupils with a scene from Figure 1 degraded at a $5 \%$ contrast.

Table 3. The contents of this table summarize the results of our calculations for a $5 \%$ contrast input scene. The columns are a descriptor for the pupil topography; fill factor by percentage of the filled aperture (note that the outermost dimension of the pupil is held equal across all pupils); the number of detected photons required to achieve a reference exposure for a filled aperture; exposure time in seconds to collect the detected photons; and the calculated exponent, $X$, in Equation 2.

\begin{tabular}{|c|c|c|c|c|c|c|}
\hline Pupil & Description & $\begin{array}{c}\text { Fill Factor \% } \\
\text { by Area }\end{array}$ & $\begin{array}{c}\text { Detected } \\
\text { Photons } \\
\text { Required }\end{array}$ & $\begin{array}{c}\text { Exposure } \\
\text { Relative to a } \\
\text { Filled Aperture }\end{array}$ & $\begin{array}{c}\text { Exposure } \\
\text { Time (sec) }\end{array}$ & $\begin{array}{c}\text { Exponent } \\
\text { X }\end{array}$ \\
\hline 1 & Filled & 100 & 23,400 & 1 & 0.0177 & - \\
\hline 2 & $\begin{array}{c}\text { Touching } \\
\text { necklace }\end{array}$ & 61.8 & 90,000 & 6.22 & 0.073 & 3.50 \\
\hline 3 & $\begin{array}{c}\text { Necklace + } \\
\text { arms }\end{array}$ & 56.0 & 100,000 & 7.63 & 0.070 & 6.00 \\
\hline 4 & Annulus & 74.2 & 104,000 & 5.99 & & \\
\hline
\end{tabular}

\subsection{The Effects of Color}

We performed a quantitative evaluation of the effects of colored object scenes and performed a limited trade-off study of color, sparse aperture configuration, detected photons, and scene contrast. This section only provides a condensed description of the analysis conducted. We found that in the presence of substantial optical bandwidth, irregular MTFs perform poorly. The MTF scales radically with wavelength. Using the MTF of one color for another color will cause improper deconvolution gain and result in color artifact. The MTF of an annulus, because it is continuous and smooth, is insensitive to bandwidth. Phase errors cannot be corrected by deconvolution in the presence of bandwidth even if the phase errors are known because phase errors at different wavelengths generate OTFs, which are very different from one another, containing many negatives.

\section{CONCLUSION}

Examining the contents of Tables 1 through 3, we conclude that for a sparse aperture telescope system designed for lowcontrast, complex-scene astronomy and planetary observations, the fill factor must be very large-at least $30 \%$ to enable imaging of $20 \%$ contrast scenes, $40 \%$ for $10 \%$ contrast imagery, and $50 \%$ for $5 \%$ contrast imagery. Also for the typical non-redundant configurations we examined, we find that very high full-well levels and long exposure times are required. There is also a tradeoff between fill factor and full-well. If fill factor is reduced then the required full-well must increase to hold a constant signal-to-noise ratio (SNR). The full-well capacity of the detector and the permissible exposure time will exceed reasonable values if the fill factor is too small. If object-space-scene contrasts fall below $5 \%$, it is not clear 
that any sparse configuration is practical. Also, in the presence of noise, a very low OTF value will appear as holes in the U-V plane. Therefore, noise will corrupt signals where the OTF is very low. No sparse systems exist capable of imaging scenes of below $3 \%$ contrast. The rationale is that below $2 \%$ contrast, even filled apertures require over 130,000 detected photons to generate poor quality imagery. This appears to be a shot noise limitation.

Image contrast is a powerful constraint, which directly limits fill factor and number of detected photons. Large fill factors and numbers of detected photons are required to achieve an SNR ratio sufficient to permit deconvolution in the presence of shot noise.

- $20 \%$ contrast requires a fill factor of $30 \%$ and 100,000 detected photons.

- $10 \%$ contrast requires a fill factor of $40 \%$ and 100,000 detected photons.

- $5 \%$ contrast requires a fill factor of $50 \%$ and 100,000 detected photons.

\section{ACKNOWLEDGMENTS}

This research was carried out at the Jet Propulsion Laboratory, California Institute of Technology, and was sponsored by the National Aeronautics and Space Administration (NASA).

\section{REFERENCES}

[1] Fienup, J. R., Griffith, D., Harrington, L., Kowalczyk, A. M., Miller, J. J., and Mooney, J.A. "Comparison of Reconstruction Algorithms for Images from Sparse-Aperture Systems," Proceedings of the SPIE 4792-01, Image Reconstruction from Incomplete Data II, Seattle, WA, (invited paper), (2002).

[2] Carpenter, K. G., Schrijver, C. J., Karovska, M., and the SI Vision Mission Team. "The Stellar Imager (SI) Vision Mission," in Advances in Stellar Interferometry, eds. J. D. Monnier, M. Scholler, and W. C. Danchi, Proceedings of the SPIE 6268-21, (2006).

[3] Carpenter, K. G., Lyon, R. G., Schrijver, C .J., Karovska, M., and Mozurkewich, D. "Direct UV/Optical Imaging of Stellar Surfaces: The Stellar Imager (SI) Vision Mission," in UV/Optical/IR Space Telescopes: Innovative Technologies and Concepts III, Proceedings of the SPIE 6687-45, (2007).

[4] Meinel, A. B., and Meinel, M. P. "Two Stage Optics: High-acuity performance from low acuity optical systems," Optical Engineering 31, 2271-2281, (1992).

[5] Fiete, R. D., Tantalo, T. A., Calus, J. R., and Mooney, J. A. "Image quality of sparse aperture designs for remote sensing," Optical Engineering 41(8), 1957-1969, (2002).

[6] Meinel, A. B., and M. P. Meinel. "Optical Phased Array Configuration for an Extremely Large Telescope," Applied Optics 43(3), 601-607, (2004). 